\title{
Kochleaimplantation bei einseitiger Taubheit?
}

Probst, R

DOI: https://doi.org/10.1007/s00106-008-1796-9

Other titles: Cochlear implantation for unilateral deafness?

Posted at the Zurich Open Repository and Archive, University of Zurich ZORA URL: https://doi.org/10.5167/uzh-4208

Journal Article

Published Version

Originally published at:

Probst, R (2008). Kochleaimplantation bei einseitiger Taubheit? HNO, 56(9):886-888.

DOI: https://doi.org/10.1007/s00106-008-1796-9 
HNO 2008 $\cdot 56: 886-888$

DOI 10.1007/s00106-008-1796-9

Online publiziert: 15. August 2008

(c) Springer Medizin Verlag 2008

\section{Redaktion}

M. Bloching, Berlin

\author{
R. Probst \\ Klinik für Ohren-, Nasen-, Hals- und Gesichtschirurgie, Universitätsspital Zürich
}

\title{
Kochleaimplantation bei einseitiger Taubheit?
}

Das Kochleaimplantat (CI) ist eine anerkannte und erfolgreiche Methode zur Habilitation oder Rehabilitation bei beidseitigen, hochgradigen und kochleär lokalisierten Schwerhörigkeiten oder Taubheiten. In den letzten Jahren etablierte sich auch die beidseitige CI-Versorgung unter bestimmten Voraussetzungen, obwohl der audiometrische Nachweis des Nutzens nicht einfach und oft anfechtbar ist [4].

Seit wenigen Jahren berichteten nun einzelne Kliniken über die Versorgung von einseitigen Taubheiten mit einem CI. Zitierbare Veröffentlichungen, die über PubMed zu erreichen wären, existieren allerdings noch nicht, die Mitteilungen beschränken sich auf Zusammenfassungen von Kongress-Beiträgen sowie Veröffentlichungen in der Laienpresse oder im Internet. Diese Mitteilungen eröffnen im Vergleich zur CI-Versorgung von beidseitig hochgradig Schwerhörigen eine Behandlung und Diskussion mit grundsätzlich neuen Dimensionen.

\section{Bisherige Erfahrungen}

Vor einigen Jahren begannen Van de Heyning, Vermeire et al. an der Universität Antwerpen ausgewählte erwachsene Patienten mit einseitig erworbener Ertaubung mit einem CI auf der ertaubten Seite zu versorgen $[8,10]$. Die Indikation dazu war immer ein Tinnitusleiden auf dem ertaubten Ohr, das auf andere Behandlungen ungenügend ansprach. Vermeire berichtete auf der Jahresversammlung der Deutschen Gesellschaft für Audiologie in Kiel am 7. März 2008 über die Erfahrung mit bisher 22 so mit einem Implantat versorgten Personen [9]. Alle Patienten tragen ihr CI tagsüber, das Tinni- tusleiden konnte bei allen günstig beeinflusst werden, und die Patienten berichten in einem entsprechenden Fragebogen (SSQ: „Speech Spatial and Qualities Hearing Scale") über einen Hörgewinn mit dem CI. Diese Befunde deuten auf eine partielle Fusion des normalen Gehörs auf der einen Seite mit dem elektrisch induzierten Hören über das CI auf dem ertaubten Ohr hin. Angesichts der grundsätzlich verschiedenen Stimulation der beiden Ohren konnte eine solche zentrale Fusion nicht ohne Weiteres erwartet werden [6]. Sie belegt erneut die erstaunlichen plastischen Fähigkeiten der zentralauditorischen Verarbeitung, wie sie schon mit der einseitigen oder beidseitigen CIVersorgung bei beidseitiger Taubheit demonstriert wurde.

Auf der Versammlung in Kiel berichteten auch Stelzig [5] und Jacob [2] sowie deren Mitarbeiter über Erfahrungen mit ähnlichen Patienten im Bundeswehrzentralkrankenhaus Koblenz. Insgesamt wurden dort seit 2005 sechs Angehörige der Bundeswehr nach erworbener einseitiger Ertaubung mit einem CI versorgt. Auch von dieser Gruppe wurde über eine subjektive Akzeptanz des CI und zudem über ein verbessertes räumliches Hören berichtet. Zusätzlich wurden in der Medizinischen Hochschule Hannover 3 ähnliche Patienten mit ähnlichem Resultat implantiert.

So gab es in Kiel Berichte über insgesamt mehr als 30 Patientinnen und $\mathrm{Pa}$ tienten mit einseitig erworbener Ertaubung und Versorgung mit einem CI, die in den letzten Jahren erfolgte. Neben einzelnen weiteren ähnlichen Versorgungen entsprach diese Zahl in etwa der vorliegenden Erfahrung mit dieser speziellen Indikation für ein CI. Sie kann aufgrund dieser Mitteilungen insgesamt als recht gut bezeichnet werden, mit wichtigen Einschränkungen wie die relative Kurzfristigkeit. Dennoch, die Patienten akzeptierten die Versorgung und scheinen einen Gewinn in der Verminderung des Tinnitus und/oder im räumlichen Hören zu haben.

\section{Offene Fragen zur Cl-Versorgung von Erwachsenen mit einseitig erworbener Ertaubung}

Tinnitus kann bei einseitig erworbener Ertaubung zu einer schwer kontrollierbaren Beschwerde werden. Die oben erwähnten Berichte deuten auf eine Verbesserung der Kontrolle und der Beschwerden durch ein CI hin. Dabei handelt es sich unbestritten um eine „Off-label-Indikation “ für das CI. Trautwein beantwortete die Anfrage einer Mutter nach einer Technologie für ihren bei einem Motorradunfall einseitig ertaubten Sohn klar in diesem Sinne [7]. Sie bestätigte die FDAAnerkennung des $\mathrm{CI}$ nur für beidseitige hochgradige Schwerhörigkeit. Sie wies zudem auf eine sehr beschränkte Erfahrung mit dem CI bei einseitiger Taubheit und auf die Notwendigkeit für zusätzliche Forschung auf diesem Gebiet hin.

In der Tat eröffnet sich mit dieser Indikation ein neues Forschungsfeld mit interessanten klinischen und grundlegenden Fragen wie:

- Ist der therapeutische Effekt des CI beim Tinnitus allein durch die elektrische Stimulation bedingt oder ist er von einer durch das $\mathrm{CI}$ erreichten Hörleistung abhängig? Im ersten Fall könnten wesentlich einfachere und billigere Stimulatoren verwendet werden, wie sie beispielsweise auch bei 
der tiefen Hirnstimulation verwendet finden, eine Behandlung, die sich in der neurochirurgischen Routine etabliert hat.

- Viele Fragen der Programmierung zur Erreichung entweder des therapeutischen Effekts auf den Tinnitus oder einer möglichst guten zentralen Fusion sind offen.

- Braucht es ein dauerndes und anhaltendes Training des tauben Ohres, um die Wirkungen des CI aufrechtzuerhalten? Die HNO-Klinik der Medizinischen Hochschule Hannover berichtete über solche Erfahrungen.

- Welche Faktoren sind mit Erfolg oder Misserfolg gekoppelt, die bei der Indikationsstellung helfen könnten?

- Wie relevant für den Alltag des Patienten ist der Zusatznutzen durch einen Gewinn an räumlichem Hören?

- Eine der wichtigsten Fragen wird den Langzeitverlauf betreffen. Werden die Patienten das CI nach 5, 10, 15 Jahren tragen? Das würde vermutlich einen entscheidenden Gewinn für die Patienten belegen.

Alle diese Fragen sind wissenschaftlich interessant, und sie sind es wert, in den kommenden Jahren mit weitsichtigen und genauen Protokollen untersucht zu werden. Nur damit wird es möglich sein, die notwendigen Entscheidungsgrundlagen für weitergehende Empfehlungen zu erarbeiten. Im Vergleich zur bilateralen CIVersorgung bei beidseitiger Taubheit erscheinen die Vorteile weniger offensichtlich und die Fachleute sind sich weniger einig.

\section{$\mathrm{Cl}$ bei kongenitaler Taubheit auf einem Ohr?}

In Kiel fand zum Thema CI-Versorgung bei einseitiger Taubheit auch eine Podiumsdiskussion statt, die als „Die Kontroverse: CI bei einseitiger Normalhörigkeit?" überschrieben war. Ich übernahm die Moderation dieses Gesprächs und begann mit der vermutlich hypothetischen Frage nach der CI-Versorgung von Kindern mit angeborener einseitiger Taubheit in der Annahme, dass eine solche Versorgung von den Teilnehmern des Rundtisches ohne viel Diskus- sion beim heutigen Wissenstand als nicht indiziert betrachtet würde. Anschließend sollte die Diskussion sich den oben beschriebenen Patienten und Fragen zuwenden. Einleitend und zur Illustration stellte ich folgenden Text aus einem Internet-Diskussionsforum der Vereinigten Staaten vor:

„My 3 year-old with Down Syndrome has unilateral hearing loss in the left ear. At his first appointment with the ENT, we were told that he would not be a candidate for cochlear implants. At a recent appoint with the Developmental Pediatrician, we were told that things have changed.“ ([1], „Mein 3 Jähriger mit DownSyndrom hat einen einseitigen Hörverlust des linken Ohrs. Bei der ersten Konsultation beim HNO-Arzt wurde uns gesagt, dass er kein Kandidat für Kochleaimplantate sei. Bei einer kürzlich stattgefunden Konsultation beim Entwicklungspädiater wurde uns gesagt, dass sich die Dinge geändert haben.") Die Frage an die Rundtischteilnehmer lautete: Hat sich die Lage wirklich verändert? Gibt es eine Indikation für ein CI bei angeborener, einseitiger Taubheit?

Im erwähnten Diskussionsforum sahen alle im Internet Antwortenden keine Indikation für ein CI bei einseitig taubem Kind. Nicht nur deshalb war ich überrascht und erstaunt, als die Mehrzahl der Teilnehmer des Rundtischgesprächs auf meine Frage sich teils deutlich für eine CIIndikation bei Kindern mit kongenitaler einseitiger Taubheit aussprach. Die Kontroverse war lanciert.

Da vor dem Rundtischgespräch günstige Resultate von Exponenten eines CI bei einseitiger Taubheit mitgeteilt wurden und solche Exponenten am Gespräch teilnahmen, mag die Runde durch die Dynamik des Augenblicks etwas weit getragen worden sein. So ist es jetzt Zeit, in Ruhe über die Implikationen einer CI-Versorgung bei einseitig kongenitaler Taubheit nachzudenken. Was gilt es zu bedenken?

Neben dem aufgrund der Erfahrung und Evidenz als klein zu bezeichnenden Risiko der Operation selbst müssen die möglichen Folgen einer lang dauernden CI-Versorgung bedacht werden. Es sind dies u. a. eine Stigmatisierung und eine Behinderung von natürlichen Kompensationsmechanismen.
Kinder mit einer einseitig angeborenen Taubheit zeigen nicht die gleiche auditorische Leistung wie Kinder mit beidseitig normalem Gehör [3]. Einschränkungen machen sich hauptsächlich im Störgeräusch bemerkbar, und diese Kinder sind insofern ohne Zweifel behindert. Die Behinderung macht sich hauptsächlich in ungünstigen Lernsituationen bemerkbar, sei dies beim Spracherwerb oder später in der Schule. Insgesamt kann die Behinderung bei der weitaus größten Anzahl von Kindern dennoch als leichtgradig bezeichnet werden, und die Kinder selbst fühlen sich in der Regel spontan nicht benachteiligt oder behindert.

Diese Bewertung wird auch durch die fast jedem HNO-Arzt bekannten Fälle von einseitigen Taubheiten unterstützt, die ohne Screening erst im Schul-, Pubertäts- oder gar Erwachsenen-Alter entdeckt wurden. Diese Kinder können eine erstaunlich gute auditorische Lokalisationsfähigkeit aufweisen. Sie nutzen dabei vermutlich diskrete Anhaltspunkte zur Lokalisation eines auditorischen $\mathrm{Ob}$ jekts wie spektrale Unterschiede und Brechungsunterschiede an der Ohrmuschel, die sich bei unterschiedlichen Lokalisationen und Verschiebungen ergeben. Solche Veränderungen der akustischen Transferfunktionen des äußeren Ohrs werden auch von Normalhörenden beispielsweise zur Abschätzung der vertikalen Lokalisation in der Frontalebene benutzt, und sie werden vermutlich von kongenital einseitig Tauben in einem wesentlich größeren Ausmaß benutzt und verarbeitet. Ihre zentrale Repräsentation könnte einen Teil des binauralen Lokalisationsverlusts wettmachen.

Diese Kinder fühlen sich nicht nur mehr oder weniger unbehindert, sie sind auch nicht stigmatisiert. Mit der CI- oder auch Hörgeräte-Versorgung werden sie aber unvermeidlich gezeichnet. Falls die positiven Auswirkungen eines solchen Stigmas die negativen klar und eindeutig übersteigen, wird eine solches Stigmatisierung medizinisch und ethisch gut zu vertreten sein. Ist diese Voraussetzung bei einer CI-Versorgung bei einseitig kongenitaler Taubheit tatsächlich gegeben?

Aus meiner Sicht fehlt Evidenz dafür oder dagegen, und es gibt weder konkrete noch intuitive Anhaltpunkte und schon 
gar keine Erfahrungen um anzunehmen, dass mit einem CI bei einseitig kongenitaler Taubheit mehr Nutzen als Schaden erzeugt wird. Negative Auswirkungen einer CI-Versorgung bei kongenital einseitiger Taubheit erscheinen neben dem eher harmlosen Stigma zumindest denkbar. Vielleicht die Wichtigste würde in der Be- oder gar Verhinderung der Ausbildung der oben beschriebenen zentralen Kompensationsfunktionen dieser Kinder liegen.

Falls das Ziel einer solchen CI-Versorgung die Ausbildung eines binauralen Richtungs- und Raumhörens ist, müsste aufgrund der vorliegenden Erfahrungen mit der CI-Versorgung bei beidseitig schwerhörigen Kleinkindern eine frühzeitige Implantation angestrebt werden, da sie am ehesten die Ausbildung einer binauralen zentralen Verarbeitung erreichen könnte. Aus dem gleichen Grund müsste das CI konsequent getragen werden. Eine frühzeitige und konsequent angewandte CI-Versorgung würde aber unvermeidlich auch die beste Phase der Bildung von Kompensationsmechanismen betreffen und sie behindern. Man gewänne mit der Versorgung möglicherweise eine Binauralität, allerdings unter Inkaufnahme einer Behinderung der monauralen Kompensationen und mit einer Stigmatisierung.

Damit ist natürlich bei der Versorgung einer postlingual einseitig erworbenen Ertaubung nicht zu rechnen, da die frühkindlich erworbenen Kompensationen ja gerade fehlen. Falls der einseitig ertaubte Erwachsene keinen Nutzen von seinem CI hat, wird er den Prozessor nicht tragen, und er wird im täglichen Leben etwa wieder am Punkt vor der CI-Operation sein. Es ist kaum ein zusätzlicher organischer und funktioneller Schaden zu befürchten. Beim frühzeitig versorgten Kind könnte aber ein späteres Nichttragen des CI zu einer Verschlechterung der auditiven Funktionen führen. Falls nämlich mit dem CI tatsächliche eine binaurale Funktion ausgebildet wäre, würde ein Nichttragen zu einer monauralen Funktion mit fehlender Kompensation führen. Bei einer permanenten Ablehnung und einem Nichtgebrauch des CI müsste teilweise von einer in Kauf genommenen erworbenen Ertaubung ausgegangen wer- den. Das Kind würde in dem Maße eine erworbene Taubheit aufweisen, in dem es Nutzen vom CI gehabt hätte.

Die Frage, ob Kinder mit einem normal hörenden Ohr ihr CI auf der Gegenseite in die Pubertät und in den nachfolgenden Lebensalter tatsächlich dauernd tragen würden, ist zumindest berechtigt. Es mag nicht zu letzt das Stigma sein, das zu den gut bekannten vorübergehenden, aber teilweise auch länger dauernden Schwierigkeiten mit CI oder Hörgeräten bei Kindern mit beidseitigen Schwerhörigkeiten in diesen Lebensabschnitten beiträgt. Falls Kinder mit einseitiger Taubheit also später beschließen, das CI wegen der Stigmatisierung oder wegen $\mathrm{zu}$ geringem Nutzen im Vergleich zum alläglichen Aufwand nicht mehr zu tragen, bliebe möglicherweise ein dauernder Schaden zurück. Mit der heutigen Kenntnis der Ablehnung in der Pubertät von Hörhilfen, deren Effizienz kaum in Zweifel zu ziehen sind, muss mit einer Ablehnung eines CI bei Kindern mit einer einseitig normalen Gehör gerechnet werden. Natürlich fehlen auch hierzu Daten.

\section{Fazit}

Die Versorgung von einseitig spät ertaubten Personen mit $\mathrm{Cl}$ verlief bis heute erfolgreich, und es ergeben sich interessante Fragen für die klinische und wissenschaftliche Forschung. Die heutigen Ergebnisse müssen aus mehreren Gründen als vorläufig und kurzfristig bezeichnet werden. Langfristige Resultate werden entscheidend sein. Im Gegensatz zu beidseitig hochgradig schwerhörigen $\mathrm{Pa}$ tienten sind einseitig Ertaubte für den Alltag nicht auf ein $\mathrm{Cl}$ angewiesen. Es wird sich zeigen, ob der Nutzen des $\mathrm{Cl}$ den damit verbundenen Aufwand auf lange Zeit rechtfertigen kann.

Ganz verschieden stellt sich die Lage für Kinder mit angeborener einseitiger Taubheit dar. Da wir nicht wissen können, was für solche Kinder auf die Dauer besser ist, eine natürlichen Anpassung an die monaurale Situation mit Kompensation oder eine mögliche, aber kaum normale binaurale Funktion mit einem $\mathrm{Cl}$, ist nach meiner Überzeugung das Risiko, mit einer Cl-Versorgung mehr Schaden als Nutzen zu erzielen, zu groß, um eine solche
Versorgung verantworten zu können. Dies ist keine wissenschaftliche, sondern eine ethische Frage. In einer solchen Situation ist es Pflicht des Arztes, auch möglichen Schaden zu vermeiden.

\section{Korrespondenzadresse}

\section{Prof. Dr. R. Probst}

Klinik für Ohren-, Nasen-, Hals- und Gesichtschirurgie, Universitätsspital Zürich, Frauenklinikstrasse 24, 8091 Zürich Schweiz

direktion.orl@usz.ch

Interessenkonflikt. Der korrespondierende Autor gibt an, dass kein Interessenkonflikt besteht.

\section{Literatur}

1. Berke J (2007) Cochlear implant with unilateral hearing loss? Answer to an About.com visitor, July 31 2007, http://deafness.about.com/ b/2007/07/31/cochlear-implant-with-unilateralhearing-loss.htm

2. Jacob R, Schleich P, Nopp P, Müller J, Helms J, Stelzig Y (2008) Cl bei einseitigen Taubheit:Wiederherstellung des Richtungsgehörs. Abstract DGA SS06, S 178, http://www.dga2008/programm/programm.html

3. Lieu JE (2004) Speech-language and educational consequences of unilateral hearing loss in children. Arch Otolaryngol Head Neck Surg 130: 524-530

4. Papsin BC, Gordon KA (2008) Bilateral cochlear implants should be the standard for children with bilateral sensorineural deafness. Curr Opin Otolaryngol Head Neck Surg 16: 69-74

5. Stelzig Y, Schleich P, Nopp P, Baumann U, Jacob R (2008) Cl bei einseitigen Taubheit: Hören im Störschall und „Pitch Matching". Abstracts DGA FV05, S 141, http://www.dga2008/programm/programm.html

6. Strutz J (2007) Welche therapeutischen Möglichkeiten gibt es heute zur Rehabilitation einer erworbenen einseitigen Schwerhörigkeit bzw. Taubheit? Schnecke 57(8): 25

7. Trautwein $P$ (2006) FDA regulations and cochlear implantation for unilateral deafness. http://www. healthyhearing.com/hearing_library/ate_content. asp?question_id=275, Stand 16.06.2008

8. Van de Heyning P, Vermeire K, De Ridder D (2007) Tinnitus treatment with cochlear implantation in unilateral sensorineural deafness. Hearing Preservation Workshop VI, Antwerp, 19.-20. October 2007, http://www.hearingpreservation.com/ shared/Abstract_book.pdf, Stand 16.06.2008

9. Vermeire K, De Ridder D, Schleich P, Nopp P, Van de Heyning P (2008) Tinnitus treatment with cochlear implantation in unilateral sensorineural deafness. Abstract DGA SS06, p 177, http://www.dga2008/ programm/programm.html

10. Vermeire $K$, Schleich $P$, Nopp $P$, Van de Heyning $P$ (2007) Bimodal hearing after $\mathrm{Cl}$ in unilateral sensorineural deafness and tinnitus. Hearing Preservation Workshop VI, Antwerp, 19.-20. October 2007, http://www.hearingpreservation.com/shared/ Abstract_book.pdf, Stand 16.06.2008 